\title{
Comparison of conventional dressings and vacuum-assisted closure in the wound therapy of Fournier's gangrene
}

\author{
Fatih Yanaral ${ }^{1}$, Can Balci ${ }^{2}$, Faruk Ozgor ${ }^{1}$, Abdulmuttalip Simsek ${ }^{3}$, Ozkan Onuk ${ }^{2}$, \\ Muammer Aydin ${ }^{2}$, Baris Nuhoglu ${ }^{2}$ \\ ${ }^{1}$ Department of Urology, Haseki Teaching and Research Hospital, Istanbul, Turkey; \\ ${ }^{2}$ Department of Urology, GaziosmanpasaTaksim Teaching and Research Hospital, Istanbul, Turkey; \\ ${ }^{3}$ Department of Urology, Bakirkoy Dr. SadiKonuk Teaching and Research Hospital, Istanbul, Turkey.
}

\begin{abstract}
Summary $\quad$ Objective: The purpose of our study was to compare Vacuum-assisted closure (VAC) and conventional dressings in the wound therapy of Fournier's gangrene (FG).

Materials and methods: The study evaluated 54 patients, retrospectively. Following initial removal of necrotic and devitalized tissue, in Group I patients the wounds were covered with conventional antiseptic dressings and patients continued to be treated with conventional dressings. In Group II patients VAC therapy was initiated. The collected data were compared between groups.

Results: The difference between two groups were statistically significant in terms of number of daily dressing (group I: 2, group II: 0,5), VAS (group I: 8, group II: 5), number of daily analgesics (group I: 4, group II: 2), number of daily narcotic analgesics (group I: 1, group II: 0), duration of mobilization per day (group I: 40, group II: 73 minutes) $(p<0.05)$.

Conclusions: Our study does not determine that a VAC therapy is better than conventional dressings in terms of clinical outcome. However, vacuum dressing appears an effective and successful method, which offers fewer dressing changes, less pain, and greater mobility comparing to conventional dressings in the management of FG patients.
\end{abstract}

KEY WORDS: Debridement; Gangrene; Prognosis; Treatment outcome; Wound closure techniques.

Submitted 26 April 2017; Accepted 2 June 2017

\section{INTRODUCTION}

In 1883, Jean Alfred Fournier described a syndrome with necrosis of the perineum in five men; this type of necrotizing fasciitis was subsequently given his name and is known as Fournier's gangrene (FG) (1). FG is a potentially life-threatening progressive infection necrotizing fasciitis of the perineal, genital, or perianal regions. It is characterized by thrombosis of the nutrient vessels leading to tissue ischemia and tissue ischemia promotes infectious dissemination leading to skin necrosis. In most cases, FG is a polymicrobial infection, with both aerobic and anaerobic organisms, which originates from a urogenital, colorectal, or cutaneous source (2).

In spite of aggressive management; it is associated with high morbidity and mortality (3-67\%) and a delay in diagnosis and treatment is known to increase mortality rates (3). Classical treatment consists of radical excision of all necrotic tissue, broad-spectrum antibiotics and intensive care. Usually repeated debridement is necessary. For this reason wounds of the patients remain open for a long time, and require frequent dressing. Different protocols have been proposed for postoperative open wound care: unprocessed honey, hyperbaric oxygenation, grown hormones, growing agents, and vacuumdressing technologies (4). Vacuum-assisted closure (VAC) device (KCI USA, Inc. San Antonio, TX USA) is a wound care system that works on the basis of negative pressure vacuuming; removes exudate and infectious materials, reduces edema and promotes healing.

The purpose of our study was to compareVAC and conventional dressingsin the wound therapy of FG.

\section{Materials AND Methods}

The study evaluated 54 patients who diagnosed of FG and received treatment between June 2001 and October 2014 at our Urology Department. All data and parameters were analyzed retrospectively. FG was diagnosed by evidence of a necrotizing fasciitisin the scrotal or perineal region. Patients with a simple inflammation without involvement of the fascia, necrotizing fasciitis at other locations and patients with incomplete clinical data were excluded from the analysis.

Intravenous replacement of fluid and electrolytes, thirdgeneration cephalosporin and metronidazole antibiotherapy were started at admission. All patients underwent surgical debridement during admission day (Figure 1). Empiric antimicrobial therapy was revised according to the results of bacterial culture and drugsensitive tests of the removed tissue samples.

Following initial removal of necrotic and devitalized tissue, in Group I patients the wounds were covered with conventional antiseptic dressings and patients continued to be treated with conventional dressings by washing repeatedly with saline until healthy granulation tissue was formed in the wound. Wound dressings were changed twice a day. After surgical debridement, in Group II patients VAC therapy was initiated. Silver nitrate sponge was used for the wounds then drape was placed over the 
Figure 1.

Patient during surgical debridement with Fournier's gangrene.

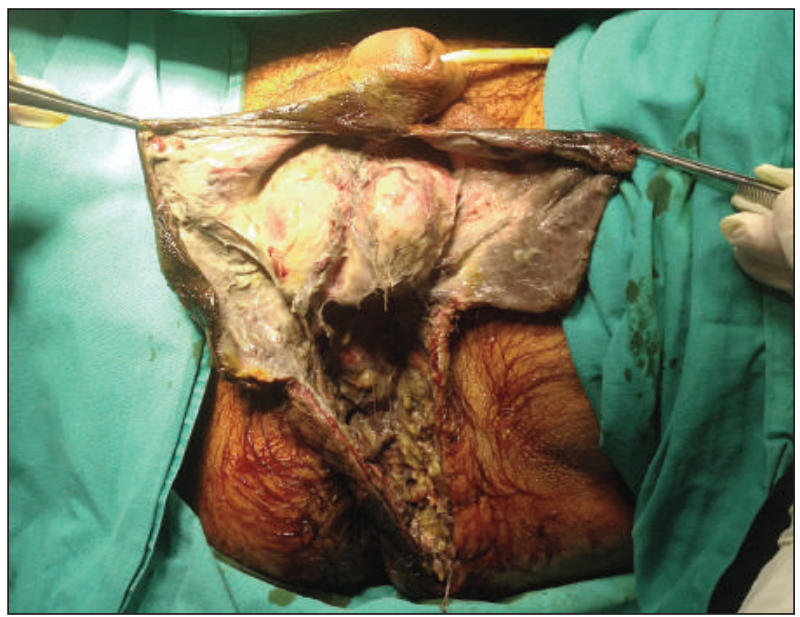

Figure 2.

VAC therapy at negative pressure.

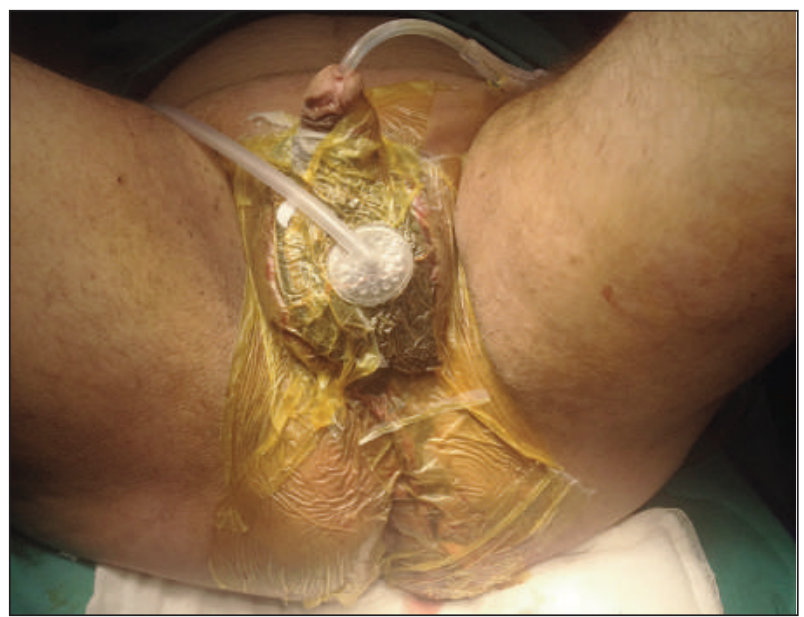

sponge, suction was inserted and continuous negative pressure was applied to the wounds (Figure 2). Initially, the pressure is set to $50 \mathrm{~mm} \mathrm{Hg}$ and increased to a maximum of $125 \mathrm{~mm} \mathrm{Hg}$. VAC dressings were changed every 48-72 hours. Additional changes were performed in both groups if the dressings became wet due to blood or fluid from the wounds. In the case of progressive necrosis, surgical debridement was repeated. After the wounds were clinically healed, in small residual defects tertiary wound closure was performed; otherwise, skin flap or graft surgery was performed (Figure 3).

We collected data on patient age, gender, history of diabetes mellitus, origin, wound diameter, duration of operation, use of VAC, the number of daily dressing, visual analogue scale for pain (VAS), need for analgesics, the duration of mobilization per day, number of surgical debridement, time from initial surgical debridement to wound closure, wound closure technique, length of hospital stay (LOS), number of deaths.

The Independent-Samples t-test, the Mann-Whitney $U$ test, chi-square test and Fisher's exact test were used for statistical analysis. Results were considered statistically significant if the $\mathrm{P}$ value was less than 0.05 .

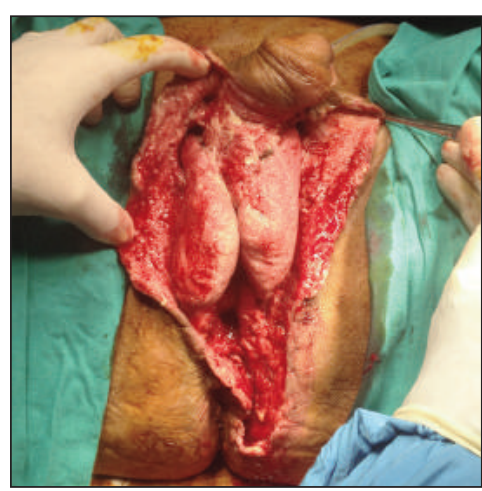

Figure 3.

Same patient as in Figure 1 and 2.

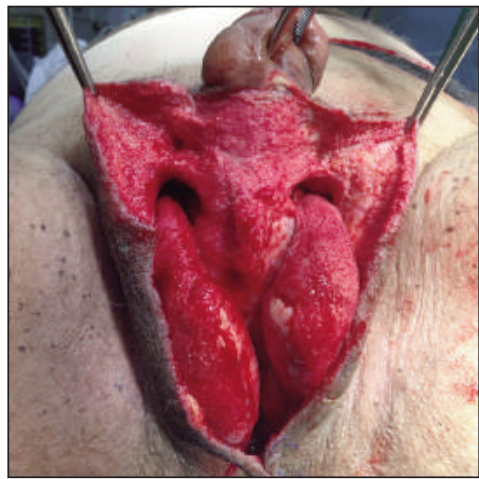

A: After the second session of vacuum therapy.

\section{B: After the third session of vacuum therapy.}

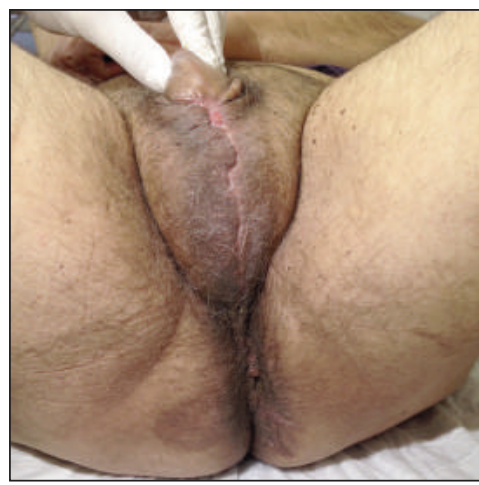

C: Three months after wound closure.

\section{RESULTS}

All the 54 patients were male, with a mean age of $55.8 \pm$ 14.9 in conventional dressings group and $61.6 \pm 7.6$ in VAC group $(p>0.05)$. Group I consisted of thirty-one patients and group II consisted oftwenty-three patients. The two groups were similar in the distribution of history of diabetes mellitus (Group I: 41.9\%, Group II: 60.9\%), wound diameter (Group I: 17, Group II: $15 \mathrm{~cm}$ ), duration of operation (Group I: 55, Group II: 48 minutes), number of surgical debridement (Group I: 1, Group II: 2), and length of hospital stay (Group I: 14, Group II: 17 days) ( $p>0.05)$. The origin of FG was anorectal diseases in 13 patients in Group I (41.9\%) and 10 patients in Group II (43.5\%). The other origin was urogenital diseases in 18 patients in Group I (58.1\%) and 13 patients in Group II (56,5\%), time from initial surgical debridement to wound closure (Group I: 12 (7-25), Group II: 13 (11-21) days) (p > 0.05) (Table 1).

However the difference between two groups were statistically significant in terms of number of daily dressing (Group I: 2, Group II: 0.5), VAS (Group I: 8, Group II: 5), number of daily analgesics (Group I: 4, Group II: 2), 
Table 1.

Preoperative characteristics of patients.

\begin{tabular}{|lccc|}
\hline & $\begin{array}{c}\text { Conventional dressings } \\
\text { group (n: 31) }\end{array}$ & $\begin{array}{c}\text { VAC group } \\
\text { (n: 23) }\end{array}$ & P \\
\hline Mean age, years & $55.8 \pm 14.9$ & $61.6 \pm 7.6$ & $>0.05$ \\
\hline DM & $13(41.9 \%)$ & $14(60.9 \%)$ & $>0.05$ \\
\hline Origin (anorectal/urogenital) & $13 / 18$ & $10 / 13$ & $>0.05$ \\
\hline Median wound diameter, cm & $17(10-45)$ & $15(9-44)$ & $>0.05$ \\
\hline DM = Diabetes mellitus. & & & \\
\hline
\end{tabular}

\section{Table 2.}

Clinical characteristics of patients.

\begin{tabular}{|lccc|}
\hline & $\begin{array}{c}\text { Conventional dressings } \\
\text { group (n: 31) }\end{array}$ & $\begin{array}{c}\text { VAC group } \\
\text { (n: 23) }\end{array}$ & P \\
\hline Duration of operation, minutes & $55(30-110)$ & $48(30-98)$ & $>0.05$ \\
\hline No. of daily dressing & $2(2-3)$ & $0.5(0.5-1)$ & $<0.05$ \\
\hline VAS & $8(4-10)$ & $5(4-10)$ & $<0.05$ \\
\hline No. of daily anelgesics & $4(3-5)$ & $2(2-3)$ & $<0.05$ \\
\hline No. of daily narcotic anelgesics & $1(0-2)$ & $0(0-1)$ & $<0.05$ \\
\hline $\begin{array}{l}\text { Duration of mobilization per day, } \\
\text { minutes }\end{array}$ & $40(0-70)$ & $73(30-120)$ & $<0.05$ \\
\hline No. of surgical debridement & $1(1-3)$ & $2(1-3)$ & $>0.05$ \\
\hline $\begin{array}{l}\text { Time from initial surgical debridement } \\
\text { to wound closure, day }\end{array}$ & $12(7-25)$ & $13(11-21)$ & $>0.05$ \\
\hline $\begin{array}{l}\text { Wound closure technique } \\
\text { (tertiary/flap or graft) }\end{array}$ & $19 / 10$ & $11 / 10$ & $>0.05$ \\
\hline \begin{tabular}{l} 
Length of hospital stay, days \\
\hline No. of deaths
\end{tabular} & $2(2-32)$ & $17(4-32)$ & $>0.05$ \\
\hline \begin{tabular}{l} 
VAS = Visual analogue scale for pain. \\
\hline
\end{tabular} & $2(8.7 \%)$ & $>0.05$ \\
\hline
\end{tabular}

number of daily narcotic analgesics (Group I: 1, Group II: 0), duration of mobilization per day (Group I: 40, Group II: 73 minutes) $(\mathrm{p}<0.05)$.

Wound closure was performed by tertiary closure in 19 and 11 patients among group I and group II, respectively. On the other hand, the wounds of twenty patients (Group I: 10, Group II: 10) were reconstructed with skin flap or graft ( $p>0.05)$. The mortality rate was lower in the group I at $6.5 \%(2 / 31)$ compared with the group II, which was $8.7 \%(2 / 23)$. But this difference was not statistically significant $(\mathrm{p}>0.05)$ (Table 2$)$.

\section{Discussion}

FG is an uncommon but life-threatening condition. Males are reported to be ten times more likely than females to develop the disease.The predisposing factors include diabetes mellitus, alcohol abuse, immunodeficiency, malignant neoplasms, and liver and renal diseases. Multiple predisposing factors represent a poor prognosis and high mortality (5). The most frequent comorbidity in patients with necrotizing fasciitis is diabetes mellitus (10-60\%). In the literature the incidence is highest in the sixth decade of life and patient age in our study groups was similar to that reported (6).

FG maybe the result of surgical wounds, skin abscess drainage, and pressure sores. It can also present as a complication of colorectal disease due to anorectal infection, ischiorectal abscesses, and colon perforations.
Other causes include a possible urethral stricture and a trauma from an indwelling Foley catheter (7). Previous studies from general surgery departments reporting perianal abscess as the most common etiological factor (8). As a urology department we found that the most common origin of $F G$ was urogenital diseases (57.4\%).

The two groups were similar in origin of disease $(p=1)$. Timing and the extent of the first debridement are the most important risk factors in terms of increased mortality rate. The relative risk of death was 7.5 times greater in cases of restricted primary debridement (9). Surgical removal of necrotic tissue caused halting the progress of the infection and eliminating the systemic effects of necrotic material, toxins, and bacteria (10).

After initial surgical debridement, management of the wound is important, along with proper nutrition of the patient. In most cases, wounds are managed with conventional dressings that contain a wide variety of active agents such as saline, povidone iodine, potassium permanganate, Dakin's solution, enzymatic agents for wound cleansing, or polyhexanide.

The other proposed protocols are unprocessed honey, hyperbaric oxygenation, grown hormones, growing agents, and vacuum-dressing technologies (4).

VAC is a device used in the general surgery, orthopedic, and gynecology in wound care management. It is also applied in the management of large wounds resulting from FG. VAC therapy has several benefits with wound area reduction and formation of granulation tissue being the most prominent. Other benefits, such as effective wound cleaning and the ability to remove the exudate render VAC a promising adjuvant therapy for wound closure (7).

Since the conventional dressings require painful changes twice a day, this has a large negative impact on the patient's quality of life. Patients in the Group II reported less pain and less need for analgesics, had greater mobility, needed fewer dressing changes than the patients in the Group I. Since patients did not need significant sedation and analgesia every day, oral intake was not limited. Thus VAC therapy can be more comfortable for patients. In a study faster discharge-using VAC device was found however, in another study no difference was reported in wound healing time comparing conventional dressing with $\operatorname{VAC}(4,11)$. In the present study, VAC therapy does not decrease wound healing time when compared with conventional dressing techniques. However, VAC effectively converts an open wound into a temporarily closed and controlled environment an it is possible to obtain much cleaner wounds without exudate by draining stagnant fluid and the debris. These devices stimulate angiogenesis and lead to an improvement of nourishment and tissue formation and create a favorable environment for healing in wound beds (12).

The length of hospitalization can be exacerbated by large tissue defects or sepsis-induced complications. The mean hospital stay was similar between group I (14 days) and in group II (17 days). The mean length of stay for all 54 patients was 16 days, which is shorter to the result reported by Czymek et al. (40 days) (13). This is due to our patients have smaller soft-tissue defects.

Death is caused by coagulopathy, acute renal failure, diabetic ketoacidosis, severe sepsis, or multi-organ failur- 
erather than local tissue defects.In the present study, there was no significant difference between the groups in mortality rate $(\mathrm{p}=1)$. Fifty of the 54 patients survived and the mortality rate of all patients was $7 \%$. The deaths of these four patients were at $6,4,2$ and 8 days. This is compatible with mortality from FG increase within the acute sepsis phase (14).

\section{Conclusions}

Our study does not determine that a VAC therapy is better than conventional dressings in terms of clinical outcome. However, vacuum dressing appears an effective and successful method, whichoffers fewer dressing changes, less pain, and greater mobility comparing to conventional dressings in the management of FG patients. The present study's outcomes should be supported by further prospective studies with a larger patient volume.

\section{REFERENCES}

1. Fournier JA. Gangrene foudroyante de la verge. Med Pract. 1883; 4:589-97.

2. Zagli G, Cianchi G, Degl'innocenti S, et al. Treatment of Fournier's Gangrene with Combination of Vacuum-Assisted Closure Therapy, Hyperbaric Oxygen Therapy, and Protective Colostomy. Case Rep Anesthesiol. 2011; 2011:430983.

3. Shyam DC, Rapsang AG. Fournier's gangrene. Surgeon. 2013; 11:222-32.

4. Tucci G, Amabile D, Cadeddu F, Milito G. Fournier's gangrene wound therapy: our experience using VACdevice. Langenbecks Arch Surg. 2009; 394:759-60.

5. Li C, Zhou X, Liu LF, et al. Hyperbaric Oxygen Therapy as an Adjuvant Therapy for Comprehensive Treatment of Fournier's Gangrene. Urol Int. 2015; 94:453-8.

6. Ferreira PC, Reis JC, Amarante JM, et al. Fournier's gangrene: areview of 43 reconstructive cases. Plast Reconstr Surg. 2007; 19:175-84.

7. Misiakos EP, Bagias G, Patapis P, et al. Current concepts in the management of necrotizing fascitis. Front Surg. 2014; 1:36.

8. Yilmazlar T, Isık Ö, Öztürk E, et al. Fournier's gangrene: review of 120 patients and predictors of mortality. Ulus Travma Acil Cerrahi Derg. 2014; 20:333-7.

9. Mok MY, Wong SY, Chan TM, et al. Necrotizing fasciitis in rheumatic diseases. Lupus. 2006; 15:380-3.

10. Vaz I. Fournier gangrene. Trop Doct. 2006; 36:203-4.

11. Czymek R, Frank P, Limmer S, et al. Fournier's gangrene: is the female gender a risk factor? Langenbecks Arch Surg. 2010; 395:173-80.

12. Cuccia G, Mucciardi G, Morgia G, et al. Vacuum-assisted closure for the treatment of Fournier's gangrene. Urol Int. 2009; 82:426-31.

13. Czymek R, Schmidt A, Eckmann C, et al. Fournier's gangrene: vacuum assisted closure versus conventional dressings. Am J Surg. 2009; 197:168-76.

14. Furr J, Watts T, Street R, et al. Contemporary Trends in the Inpatient Management of Fournier's Gangrene: Predictors of Length of Stay and Mortality Based on Population-based Sample. Urology. 2017; 102:79-84.

\section{Correspondence}

Fatih Yanaral MD (Corresponding Author)

fyanaral@yahoo.com

Faruk Ozgor, MD

md.farukozgor@gmail.com

Haseki Training and Research Hospital, Urology Department

Millet Street, Fatih, Istanbul (Turkey)

Can Balci, MD

mbcbalci@gmail.com

GaziosmanpasaTaksim Training and Research Hospital,

Urology Department KarayollariMah. Osmanbey Cad. Gaziosmanpasa,

Istanbul, Turkey

Ozkan Onuk, MD

drozkanonuk@gmail.com

Muammer Aydin, MD

maydinmd@yahoo.com

Baris Nuhoglu, MD

drbnuhoglu@gmail.com

Abdulmuttalip Simsek, MD

simsek76@yahoo.com

Bakirkoy Dr. SadiKonuk Training and Research Hospital,

Urology Department ZuhuratbabaMah. TevfikSaglam Cad. Bakirkoy,

Istanbul, Turkey 